\title{
e-Phaïstos
}

e-Phaïstos

Revue d'histoire des techniques / Journal of the history

of technology

VII-1 | 2019

Le travail humain

\section{Les sciences du travail face aux capacités des invalides}

Épistémologie, élaboration politique du travail et anthropologie, 1910-1930

Labor Sciences and Abilities of Impaired People: Epistemology, Political

Construction of Work and Anthropology, 1910-1930

\section{Stéphane ZYGART}

\section{OpenEdition}

Journals

Édition électronique

URL : http://journals.openedition.org/ephaistos/4505

DOI : $10.4000 /$ ephaistos. 4505

ISSN : 2552-0741

Éditeur

IHMC - Institut d'histoire moderne et contemporaine (UMR 8066)

Référence électronique

Stéphane ZYGART, "Les sciences du travail face aux capacités des invalides », e-Phaïstos [En ligne],

VII-1 | 2019, mis en ligne le 06 avril 2019, consulté le 19 avril 2019. URL : http://

journals.openedition.org/ephaistos/4505; DOI : 10.4000/ephaistos.4505

Ce document a été généré automatiquement le 19 avril 2019

Tous droits réservés 


\title{
Les sciences du travail face aux capacités des invalides
}

\author{
Épistémologie, élaboration politique du travail et anthropologie, \\ 1910-1930 \\ Labor Sciences and Abilities of Impaired People: Epistemology, Political \\ Construction of Work and Anthropology, 1910-1930
}

Stéphane ZYGART

1 La science du travail, telle qu'elle s'est développée au début du XXe siècle en France avec Armand Imbert, Jean-Maurice Lahy et Jules Amar est mise à l'épreuve par les invalides de la Première Guerre mondiale ${ }^{1}$. En effet, le projet général de cette science est de parvenir à une juste mesure des capacités de travail de tous, calculées sur une base psychophysiologique, afin que chacun puisse travailler dans la mesure de ses capacités et ainsi ne soit pas épuisé à la tâche tout en accomplissant son devoir social. Dans cette perspective, chaque individu est un "moteur humain ${ }^{2}$ " susceptible d'une certaine activité : l'invalidité n'y change rien. Tout invalide est, à la limite, du simple fait qu'il est vivant, capable d'une certaine activité calculable et utilisable dans un cadre professionnel.

Or, Amar le reconnaît explicitement et sans aucune ambiguïté pendant la guerre : parmi les invalides de guerre, il y en a $20 \%$ qui ne peuvent retourner à l'emploi ${ }^{3}$. Lahy évoque quant à lui les « déchets sociaux» que doit mettre de côté la sélection professionnelle ${ }^{4}$. Pourquoi ? Ni lui ni Amar ne s'étendent sur ce point. Parce que les capacités des invalides sont trop faibles - sans aucun doute - et qu'elles ne permettent pas d'atteindre (selon Amar) le "rendement social minimal qui seul [le] préoccupait ${ }^{5}$. Mais qu'est-ce qui provoque cette faiblesse qui fait rupture et seuil ? Amar, qui ne craint pas de promettre un rendement digne d'emploi à des amputés d'un bras, désespère par contre du cas des doubles amputés, mais aussi des aveugles privés de la capacité de mise en relation que permet la vue. À la même époque pourtant, d'autres - médecins, rééducateurs, philanthropes - songent pour les aveugles à des professions de vannerie, brosserie, etc. Et, en dehors des appréciations différentes des incapacités provoquées par telle ou telle 
blessure, les raisons pour lesquelles ces incapacités ont pour conséquence l'exclusion du monde du travail ne sont absolument pas mentionnées par Amar. S'agit-il d'un problème de fatigue trop rapide, ou d'une absence trop grande de capacité ? Amar affirme que les «blessés graves» "ne disposent plus d'une capacité de travail utilisable ou fort peu ». Mais alors, si l'improductivité est en cause, celle-ci doit-elle être reliée au socioéconomique? Et si oui, comment? On ne le saura pas précisément, ni chez Lahy, même si celui-ci ne se limite pas à considérer les « moteurs humains".

Tous deux semblent reconnaître une exclusion de fait de certains individus de l'emploi, au sujet de laquelle la science prolixe qu'ils déploient par ailleurs ne dit pas grand chose alors même que ce «fait » n'est pas compatible d'évidence avec les théories du moteur humain, et qu'il aurait mérité sans doute, au minimum, quelques justifications plus étendues.

4 S'agit-il d'une limite de la science du travail basée sur l'étude de la psychophysiologie du travail ? Et si tel est le cas, cette limite témoigne-t-elle d'une lacune des principes qui y étaient à l'œuvre? Ou bien cette limite est-elle extérieure à cette science, obéissant à d'autres principes (économiques, sociaux, politiques) ? Cependant, dans ce dernier cas, pour quel motif alors ces autres principes - qui sont pourtant de poids en matière d'emploi - pourraient-ils être considérés comme étant en dehors de la science du travail qu'il s'agit de construire? Quelle que soit l'option choisie, le «fait» de l'exclusion de certains invalides $\mathrm{du}$ monde professionnel menace le projet d'une science de l'organisation du travail qui serait fondée sur la détermination des capacités psychophysiologiques de tout un chacun. Mal intégré aux raisonnements de cette science, ce « fait » témoigne d'une incapacité d'appréciation de certains facteurs de l'organisation du travail - qu'ils aient été mal compris ou tout simplement ignorés (comme on le verra, Amar et Lahy ne sont pas du tout identiques sur ce plan). La bordure de la science psychophysiologique du travail peut bien être dans l'épistémologie des corps ou dans l'économie politique : dans tous les cas, elle menace cette science à la fois en tant que science et en tant qu'horizon politique où chacun, travaillant à sa juste mesure, participerait à l'harmonie sociale.

5 Pour éclaircir les problèmes posés par les invalides à cette science du travail, leur signification et leur portée, on se propose de repartir ici des difficultés précises qui furent rencontrées avec eux et des controverses que celles-ci ont pu provoquer. Plutôt que de s'interroger immédiatement sur les rapports entre science et socio-politique à partir de cette science du travail, on voudrait d'abord voir en quoi les invalides - parce qu'ils sont un cas extrême pour cette science du travail - expriment d'abord quelque chose qui est en même temps scientifique et politique, et dont l'articulation reste à modéliser.

\section{Les trois difficultés posées par les invalides de guerre à la science du travail}

6 L'appréciation des capacités de travail des invalides de guerre - seuls infirmes dont le nombre et le sort justifiaient dans les années 1910-1930 un tel essai - s'est heurtée à trois écueils principaux: le caractère incalculable de la puissance d'agir d'abord, où l'évaluation de la fatigue se noue de manière confuse à la spontanéité ou à la contrainte qui provoque l'activité ; la valeur de la modélisation a priori des procédures de travail ensuite, où l'endurance possible dans le travail que cette modélisation permet ne garantit 
pas forcément la productivité et la motivation nécessaires ; enfin, la possibilité d'intégrer les invalides à une production collective alors que les principes d'organisation de cette dernière ne se prêtent guère à une approche individualisée des travailleurs.

7 L'incalculable, le non-modélisable, l'inorganisable sont donc les trois obstacles incarnés par les invalides aux visées de la science du travail telle que la recherchent Imbert, Amar et Lahy. Tous ces obstacles ne sont pas décisifs de la même manière, et les critiques auxquelles ils ouvrent peuvent être plus ou moins connues et prévues par ces derniers. De même, ces critiques peuvent être explicitement adressées au projet d'une science psychophysiologique du travail, ou bien se trouver - plus simplement mais aussi plus radicalement - exprimées par l'indifférence des pratiques. Il faut ainsi faire attention dans chaque cas à deux choses: des solutions aux difficultés posées par les invalides étaient-elles prévues ou non? Dans quelle mesure et pourquoi n'ont-elles pas été mises en œuvre?

\section{Comment calculer la puissance de travail : psychologie et normativité}

8 Soit le calcul de la puissance de travail. Les problèmes ne furent pas là causés par la singularité ou l'étrangeté des mutilés de guerre - comme on pourrait le croire au premier abord. Ils ne résidèrent pas non plus dans l'évaluation des capacités à faire tel ou tel acte. Si cette évaluation n'était pas toujours évidente, les grandes lignes de récupération possible des capacités fonctionnelles (avec ou sans prothèses) étaient formulées, notamment par Amar'. Ce qui était en question, c'étaient deux extrêmes par rapport à la capacité d'un agir ponctuel: la détermination de la pure et simple capacité d'agir en général, d'une part, et l'appréciation des capacités à mener une activité de longue haleine, d'autre part.

Quel est l'état qui permet à une personne un agir minimal ? Pendant la guerre, à la surprise des médecins des hôpitaux, leurs pensionnaires s'étaient d'eux-mêmes remis au travail sur leur lit d'hôpital pour échapper à l'ennui, dans une sorte de naissance spontanée du travail occupationnel. Cette surprise fut rapidement mise à profit par les médecins, qui usèrent de cette activité spontanée à des fins thérapeutiques, en mettant en œuvre une ergothérapie improvisée - bien que le terme n'existât pas encore ${ }^{7}$. Sur cette base, un emballement se donne à lire dans les archives. La mise en activité des soldats convalescents devient une sorte de panacée, dont on soutient qu'elle est à même de favoriser des guérisons de blessés graves dont l'état n'est pas encore stabilisé. En 1919, on écrit ainsi - alors que la guerre est passée - que « les amputés de l'avant-bras peuvent reprendre quelques travaux peu de temps après l'amputation. [...] Il serait à souhaiter [...] que très tôt après l'amputation d'un bras (15 à 20 jours par exemple) le blessé puisse travailler une heure par jour dans un atelier. Son moignon se formerait et gagnerait en puissance et en mobilité et le mutilé reprendrait rapidement puissance et courage $»^{8}$. Aucune limite ne semble pouvoir être posée à la réduction des périodes de convalescence sans travail puisque, en 1919 encore, on explique dans une thèse de médecine sur La rééducation professionnelle des amputés des deux bras que "l'expérience a appris [..] que l'envoi hâtif aux champs des blessés qui n'intéressent plus le chirurgien pouvait être poussé plus loin qu'on ne l'avait cru $»^{9}$. Le cas le plus frappant est sans doute celui de la 
mise au travail des paraplégiques, mené à titre expérimental puis systématisé par le Docteur Camus ${ }^{10}$.

Deux confusions opèrent : entre la santé et l'activité, d'une part, et entre l'activité et le travail, d'autre part. L'activité en vient à être considérée par les médecins à la fois comme un signe de santé et comme un facteur de santé à venir. Être actif, c'est être en bonne santé ; être actif, c'est recouvrer la santé. Très vite, il apparaît légitime de pousser à l'activité des malades de plus en plus faibles, sans aucun repère sanitaire strict. Et, dans le même moment, il apparait utile et tout à fait justifié de faire de l'activité des malades l'occasion d'un travail productif - quitte à user de contraintes, les paraplégiques oisifs étant, par exemple, promis à l'hospice à la différence des autres.

On peut saisir à partir de là les problèmes posés à l'autre extrême par les travaux de longue durée avec les mutilés - problèmes plus unanimement reconnus que les précédents. D'abord, tout comme la limite inférieure de la mise au travail s'échappe, on ne parvient pas à saisir de quels efforts les soldats blessés sont réellement capables. En 1923, des médecins rééducateurs écrivent en ce sens : « Nous pensions autrefois, d'après les observations faites sur les mutilés, que, sous l'influence de la nourriture plus abondante, de l'entraînement progressif aux exercices manuels, de la vie régulière, les malades tels que les entéritiques, les cardiaques et les tuberculeux osseux, même assez déprimés, se remonteraient à l'école, nos prévisions n'ont pas été réalisées; aussi ne recevons-nous plus actuellement que ceux que nous jugeons susceptibles de résister au régime scolaire ${ }^{11} »$. L'inconnu ne se rencontre pas seulement avec les malades. Des anciens combattants à l'état de santé pourtant stabilisé ne tiennent pas le travail. « La rééducation toute physique ne suffit pas pour qu'un mutilé trouve dans les ateliers un emploi normalement rémunéré. Il faut encore que ce mutilé ait conservé une mentalité qui lui fasse admettre la discipline ; qu'il soit régulier dans ses présences, consciencieux dans son travail. Sinon l'industriel ne trouvera en lui qu'un élément de trouble, de gâchis, et malgré tout son bon vouloir, ne pourra le maintenir dans les Ateliers. [...] Et, malheureusement, il semble, d'après quelques cas connus de nous, que parmi les Mutilés qui ne sont pas encore placés ou qui se plaignent de leurs désillusions, le mal soit surtout là. Ce n'est pas que nous ne concevions que ceux qui souffrent moralement et physiquement de leur mutilation ne puissent pas être naturellement aigris $[. . .]^{12} »$. La motivation joue, c'est-à-dire des facteurs psychologiques qui échappent aux grilles d'évaluation de l'époque.

Par rapport à toutes ces difficultés, la science du travail d'alors avait des choses à dire. Il semble que qu'elle n'ait pas été considérée et qu'elle aurait dû l'être. Dès Imbert, la détermination des signes de fatigue avait été le cœur des recherches. Empêcher l'épuisement dans le travail était la garantie de l'harmonie sociale, et cela justifiait toutes les quantifications à faire ${ }^{13}$. En 1918, Lahy consacre un article entier à la fatigue psychologique : Comment peut-on déterminer scientifiquement la fatigue chez les ouvriers qui n'accomplissent pas d'efforts musculaires ${ }^{14}$ ? Amar et lui s'accordent sur bien des points à ce sujet. Il existe des signes objectifs de toute fatigue que l'on peut déceler par la modification des relevés graphiques de l'activité, des échanges respiratoires, du rythme cardio-respiratoire, etc. La fatigue psychologique se traduit de la sorte par l'augmentation de la pression sanguine ou de la température du corps ${ }^{15}$. Pour les deux hommes, le recours à l'expertise scientifique est sur cette question incontournable : car on peut mentir sur sa fatigue ou s'illusionner à son sujet alors que l'effort poursuivi en état de fatigue peut produire des dégâts irréversibles bien qu'inaperçus sur le moment. 
13 De tout cela, on ne trouve nulle trace dans les textes laissés par les médecins et les éducateurs qui agissaient au quotidien auprès des mutilés. L'urgence, le contexte, les préjugés semblent avoir tout écrasé dans la fausse évidence du faisable. Mais l'opposition qui se dessine entre science de laboratoire et pragmatisme ignorant du quotidien est trompeuse. Amar avoue son impuissance à saisir objectivement l'impact de la psychologie sur l'énergétique du moteur humain. Si la volonté et la sensibilité peuvent être approchées par l'étude des temps de réaction et qu'elles appartiennent à la physique du système nerveux, le coût et les effets de l'intelligence sont, par contre, insaisissables. Amar hasarde une vague hypothèse de radioactivité à ce sujet ${ }^{16}$. Lahy est plus circonspect encore. Passées les fonctions intellectuelles inférieures - mesurables par exemple via le temps de réaction -, aucune quantification ne lui semble possible ${ }^{17}$. Surtout, à ses yeux, des facteurs sociaux peuvent jouer - parmi lesquels la contrainte hiérarchique, qui peut amener à masquer ou à ignorer inconsciemment la fatigue ${ }^{18}$.

Ce n'est donc pas seulement que les recherches des psychophysiologues aient été négligées au sujet de l'endurance des invalides de guerre, alors qu'elles auraient pu garder de certaines dérives et lever des incertitudes. Les zones d'indétermination sont aussi les mêmes pour Amar, Lahy et les rééducateurs. Ce que l'esprit - en un sens psychologique mais aussi psycho-social - est capable de faire faire ne peut s'anticiper ou se mesurer avec certitude. Sans doute, Lahy et Amar auraient été plus méthodiques que les rééducateurs : ils se seraient par exemple méfiés des effets de l'émulation ou de la contrainte. Mais fallait-il empêcher les convalescents de s'activer et d'éprouver leurs forces dans des efforts potentiellement excessifs - ainsi que l'ont fait les soignants sur le terrain? Rien n'est moins sûr ${ }^{19}$. L'activité du vivant, en tant qu'adaptation ou réadaptation, suivant l'idée canguilhémienne de normativité, ne peut être un simple fonctionnement. Elle doit aussi nécessairement être capacité de transformation de ses propres normes par le vivant en étant capacité de transformation de son milieu, ce que n'ont peut-être pas assez considéré les psychophysiologues du travail du début du $\mathrm{XX}^{\mathrm{e}}$ siècle. "Toute normalité ouverte sur sa correction éventuelle possible est normativité authentique, est santé. Toute normalité fermée sur le souci de son maintien, hostile à toute variation des termes qui l'expriment, incapable d'adaptation à des situations nouvelles est une normalité déshabitée par l'intention normative ${ }^{20} »$. La normativité implique l'expérience de l'excès possible et la possibilité de s'y maintenir ou de s'en remettre, expérience qui est celle du vécu de la santé et celle du vécu des guérisons.

En ce sens, relativement à la mesure des capacités des mutilés de la Première Guerre mondiale, l'ignorance des sciences du travail de l'époque recoupe l'absence de repères des médecins et des rééducateurs qui se passent de ces sciences. La modélisation des processus de travail ouvre à d'autres problèmes et à d'autres limites.

\section{Faut-il passer de l'agir au travail ? L'enjeu des combinatoires fonctionnelles expérimentales}

16 Sur cette question, le discours des médecins rééducateurs est marqué par les possibilités d'intervention et de combinatoires infinies par lesquelles les mutilés peuvent reconquérir leurs capacités de travail perdues. La singularité des blessures l'explique en grande partie. Ainsi que l'écrit le Docteur Bourillon : «L'instruction qu'on leur donne [aux mutilés ] doit naturellement suivre une méthode différente de celle qui s'applique aux valides. On 
se trouve en face de difformités variées qui obligent à l'individualisation de cette méthode $\aleph^{21}$. Faute de pouvoir mettre en œuvre une imitation devenue impossible, les mutilés doivent créer eux-mêmes leurs propres manières de faire. L'idée était déjà formulée avant-guerre, comme en témoigne cette préconisation de 1910 au sujet des accidentés du travail : « Un ouvrier normal ne peut évidemment indiquer à un amputé de plusieurs doigts de chaque main comment il doit s'y prendre pour faire une brosse, coller une boîte ou relier un livre. Il ne peut qu'exécuter le travail devant l'élève mutilé qui s'ingénie selon son intelligence et les mouvements qu'il peut exécuter avec ses doigts et ses moignons ${ }^{22}$ ». C'est d'ailleurs ce qui pousse les rééducateurs à jouer sur l'émulation entre les mutilés, et à affirmer que : "Le maître mutilé serait l'idéal. Outre l'exemple vivant qu'il donnerait, il improviserait des techniques ou même seulement des façons nouvelles, dont un valide ne soupçonne pas la portée et l'utilité pour les infirmes ${ }^{23}$ ». L'empirisme de la méthode ne dépend donc pas seulement des circonstances de la guerre et d'une urgence sans moyens. Elle s'appuie sur trois piliers beaucoup plus théoriques et pérennes : la variation possible des projets avec des mutilations similaires où s'exprime la volonté des blessés, la multiplicité des compensations possibles en fonction des savoirs et des savoir-faire antérieurs des individus, l'infinité des usages possibles d'objets par lesquels passer pour contourner une déficience, puisque le corps n'est pas isolé mais nourri de son environnement.

Amar en particulier s'inscrit totalement contre cette manière de voir, et ses positions divisent les rééducateurs eux-mêmes durant le conflit ${ }^{24}$. Ses arguments sont de deux ordres, où le second gouverne le premier. D'abord, selon lui, laisser libre cours à l'invention des mutilés donne facilement un résultat, mais sans indiquer du tout que ce résultat puisse être le meilleur possible. Collé au présent, l'empirisme ne permet pas de déterminer le meilleur avenir possible. Or celui-ci doit reposer sur le maximum d'utilité possible pour le mutilé, sans épuisement: l'argument de l'endurance dans l'activité (caractéristique de tout travail) gouverne celui de l'anticipation de l'avenir le plus optimal $^{25}$.

S'exprime là l'idée selon laquelle le travail est une activité que l'on doit pouvoir répéter durablement sans fatigue. Cette idée guide toutes les recherches d'Amar et de Lahy. Elle témoigne de la subsomption de leur conception de l'activité sous l'exigence d'un travail comme activité continue, réitérée et productive. Amar s'oppose ainsi, certes, aux rééducateurs au sujet de l'usage irremplaçable de la science, seule garantie pour lui de pratiques solides. Mais à l'arrière-plan de ce désaccord joue aussi autre chose que la question de la nécessité de la théorie pour fonder la pratique. Le problème est de savoir si toute activité possible est aussi une activité valable - et donc de savoir ce qui fonde la valeur de l'activité. Face à des individus traumatisés, irrémédiablement diminués, la plupart des rééducateurs de terrain se satisfont de la reprise d'un travail, quel qu'il soit. Amar, quant à lui, espère en la restauration des capacités - le « rendement normal » - et en un avenir professionnel standard.

19 Au-delà de l'abstraction dont on a pu l'accuser, il cultive ainsi un double optimisme qui est aussi reconnaissance d'un double impératif, très incertain. La croyance dans le retour méthodique à l'emploi repose sur le caractère désirable de celui-ci pour la société et pour les mutilés. Or il n'est pas sûr du tout que la reconquête de leur possibilité d'agir par les anciens combattants corresponde à une volonté de retourner à un travail productif ${ }^{26}$. Le problème est, sur ce point, socio-politique, et il touche à un présupposé des sciences du travail qui commande leur déploiement et qu'Amar n'interroge, pour sa part, pas du 
tout: celui de l'identification de l'activité au travail. Une valeur - celle du travail structure sur ce point les recherches d'Amar et de Lahy dont leur science est l'énoncé des conditions de réalisation, et ne saurait donc être uniquement la description d'une objectivité.

Il n'en reste pas moins qu'une fois cette centralité du travail admise, les méthodes préconisées par Amar et Lahy semblent parfaitement remplir leur office: organiser efficacement le travail en conciliant productivité et bien-être de la main-d'œuvre. Or, en réalité, sur ce point - encore plus que les autres, peut-être -, elles échouent également, et il faut s'attacher à comprendre pourquoi.

\section{Pourquoi faire évaluer des individus ? Particularité pathologique et organisation du travail}

21 Amar et Lahy défendent tous deux une évaluation individualisée des capacités de travail. Il faut examiner chaque personne à part et, au sujet des invalides de guerre, Amar insiste même sur la courte durée de l'examen d'une heure comparativement aux profits qu'on peut en tirer ${ }^{27}$. Seule la connaissance précise de la réalité peut permettre de donner à chaque travailleur sa bonne place. Pour cette raison, Amar et Lahy insistent sur la nécessité des relevés graphiques de l'activité en temps réel et n'accordent pas de crédit aux courbes fonctionnelles obtenues par extrapolation de relevés ponctuels ${ }^{28}$.

Selon Amar, cette méticulosité des évaluations doit avoir un double effet social. L'employé tire une sécurité de la connaissance de sa valeur - non seulement parce que celle-ci est scientifique, mais aussi parce que cette scientificité fonde la valeur qu'on accorde à l'employé dans la durée. Celui-ci se trouve alors «déchargé de ce poids obsédant : le souci de l'avenir. La science a opéré en lui une transformation morale des plus heureuses qu'il ne cherche pas à dissimuler ${ }^{29}$. L'employeur est rassuré également parce qu'il peut prévoir la « valeur sociale exacte » de ses employés, tandis qu'il sait qu'il tirera le maximum des ouvriers " éduqués pour servir avec un maximum de rendement ». Amar propose ainsi l'établissement de fiches d'aptitudes - comprenant des informations sur la blessure, la perte de rendement, la famille, etc. - établies dans les écoles de rééducation ${ }^{30}$. Certains le suivront dans ce projet, comme Mazel qui envisage en 1917 de systématiser ces fiches dans les usines, en les faisant signer par l'employeur, l'ouvrier et le médecin, et en y ajoutant des mesures très précises - articulatoires, circulatoires, musculaires, etc. qui courent sur quatre pages ${ }^{31}$. D'autres essaieront d'élaborer (en s'inspirant d'Amar) des méthodes alternatives pour évaluer les capacités de travail des mutilés de guerre, en essayant de concilier l'inventivité de ces derniers (à l'encontre d'Amar) et la rigueur des calculs. Il faut par exemple procéder en cinq temps selon Daussat: observer anatomiquement et fonctionnellement l'ouvrier invalide, observer le même travail chez les valides, comparer les rendements, voir si une éventuelle différence de rendement s'explique médicalement, et sinon faire appel à l'ingénieur comme expert technique pour essayer de l'expliquer ${ }^{32}$.

Ces fiches et ces méthodes d'évaluation ne seront jamais significativement mises en œuvre. Les examens, les comparaisons à faire pour saisir la singularité des mutilés sont trop lourds relativement à ce qu'exige la concurrence ; les fiches reposent sur un idéal de confiance et de transparence éloigné de la réalité des rapports sociaux de travail, et en particulier du rapport entre le travail et les mutilés de guerre. Ces derniers ne 
représentaient que peu d'intérêt d'un point de vue productif, tandis que le coût de leur assistance était moindre que prévu à la suite de leur retour chez eux et des secours de leurs familles - les œuvres de guerre, dont les institutions de rééducation cessèrent d'être soutenues par l'État dès janvier 1919, ce qui favorisa leur fermeture ${ }^{33}$. Quelques très rares plaintes sur les aménagements de leur travail se trouvent dans les périodiques édités par les mutilés, sur la difficulté pour les manchots, par exemple, de se saisir des gros volumes ou de tenir les pages sur lesquelles il écrit ${ }^{34}$. Mais elles restèrent sans écho, tandis que les mutilés eux-mêmes étaient enclins au silence ou à la retraite sociale ${ }^{35}$. Pour toutes ces raisons, il n'y eut ni évaluations quantitatives, ni évaluations qualitatives rigoureuses du travail des mutilés et l'empirisme fut de mise, ainsi qu'il s'exprime dans les répertoires épais et confus des métiers ouverts aux mutilés en fonction de leurs blessures ${ }^{36}$.

L'explication de l'échec des visées d'Amar - et de celles de Lahy, qui le suivait au moins sur ce point de l'individualisation des évaluations - semble évidente: ils ont négligé l'organisation socio-économique du travail, dont ils ne parlent quasiment pas et sans rien en dire d'original en matière de débouchés ou de techniques de placement des invalides ${ }^{37}$. Cette mise de côté saperait l'intégralité de leur projet dans un oubli sans remède, beaucoup plus grave que les incertitudes liées à la fatigue ou qu'à la valorisation implicite du travail qui structure leur discours. Inconséquence scientifique et utopie politique seraient ici les deux faces d'une même pièce. Et il serait difficile de se prononcer sur l'origine de l'erreur: est-ce l'inattention au socio-économique qui aboutit à faire une science incomplète, et donc fausse? Ou bien est-ce une trop grande confiance dans la régulation sociale par la connaissance des corps qui mène à la mise entre parenthèses des autres règles possibles de l'existence sociale (domination, exploitation, menace, etc.) ? Un examen attentif des positions de Lahy montre que l'origine des difficultés n'est en réalité ni scientifique, ni politique, mais qu'elle est commune à ces deux domaines et réside dans une affirmation anthropologique de départ sur l'homo laborans, que la situation des mutilés et leurs réactions d'après-guerre remettent directement en cause.

\section{Anthropologie, science et politique du travail}

Cette position anthropologique se trouve exprimée avec force par Lahy à la fin de son livre consacré à la critique de Taylor: Le système Taylor et la physiologie du travail professionnel. On peut croire, à partir de leurs positions communes, qu'elle était partagée par Amar et Imbert sur un point essentiel : celui de la spontanéité des êtres humains à travailler.

Lahy multiplie dans cet ouvrage les critiques scientifiques, opposant les courbes de Taylor aux relevés graphiques de l'activité, l'ignorance (par Taylor) des temps de repos à la possibilité d'un refoulement de la fatigue par les ouvriers, les effets momentanés de cette fatigue à ses effets sur le long terme, la sélection locale des meilleurs (que l'on prend comme étalons) au calcul rigoureux des capacités de travail, les calculs de vitesse faits en vue d'un maximum de rendement aux calculs globaux d'une fabrication qui permettent de fixer le prix de revient ${ }^{38}$. Tout peut se résumer à un antagonisme entre la recherche d'un maximum par une approche tendancielle sans possibilité de donner une assise aux limites constatées (Taylor) et la fixation d'un optimum par un ensemble de mesures ancrées dans la science des corps (Lahy). 
mauvaises méthodes de calcul. Les attaques portent sur la façon dont il faut concevoir les individus au travail : " Taylor, avons-nous dit, a voulu construire dans l'abstrait un ouvrier-type, travaillant dans une usine-type avec des outils-types. Or, cet ouvrier-type ne correspond pas à l'idée que nous nous faisons du travailleur moderne: intelligent, actif, plein d'initiative, créateur dans le cercle de ses compétences. L'ouvrier suivant les données de W. Taylor n'est que le manouvre. C'est bien, en effet, à une dépréciation de l'ouvrier qualifié qu'aboutit le système ${ }^{39}$ ». Taylor entend obtenir des ouvriers un maximum de rendement, sous contrainte de la comparaison et de l'organisation des tâches, en partant du principe que ce maximum - et même la fiabilité des relevés - ne peuvent être garantis à cause de la paresse potentielle des travailleurs. Lahy, au contraire, défend une spontanéité et une bonne volonté initiale des personnes dans le travail qui rend inacceptable à ses yeux les dégâts du travail sous contrainte. D'autre part - et c'est le point sur lequel Lahy se sépare d'Amar tout en s'opposant à Taylor - ce rapport positif au travail est fondé en dernière instance sur une spontanéité à l'activité en général - activité libre qui ne prend pas forcément la forme d'un travail (jeu, essai, etc.), mais qui peut aussi la prendre. Par conséquent, pour Lahy, il faut également penser l'activité sociale hors travail pour penser le travail - ce qui change considérablement l'objet de la science du travail et les variables de celle-ci. L'organisation du travail doit se faire à partir de la transformation de l'activité spontanée en travail : il faut donc intégrer dans la pensée du travail le hors-travail comme condition de possibilité du travail.

Lahy ouvre ainsi ses réflexions, comme Imbert avait pu le faire ${ }^{40}$, au syndical et à la vie socio-politique au sens large, le lieu de naissance des formes de travail, lesquelles en retour modèlent nos manières d'agir. La fabrication spontanée d'objets par les combattants au front dans une reprise rudimentaire des formes sociales normales de travail - que Lahy avait pu relater en septembre 1915 - prend place dans cette théorisation ${ }^{41}$. Peut-on dire alors que ce socio-politique est scientifiquement saisi par Lahy, et pour quelles conséquences? Il n'est qu'indiqué par lui, en bordure de ses textes et de ses relevés scrupuleux, et pas du tout formellement exposé comme le reste. Plutôt que d'y voir un défaut, on devrait peut-être y déceler la rigueur épistémologique de Lahy : à objets différents (le corps, le socio-politique), discours différents. Et surtout, puisque Lahy fait un pari sur la spontanéité et l'intelligence, n'est-il pas légitime que la source du travail qui se fait au dehors des usines n'ait pas d'autres formes que celle de l'invention qu'on peut signaler, espérer, que les mutilés n'étaient peut-être que peu enclins à poursuivre, mais qu'il serait en tous cas contradictoire de vouloir modéliser en dehors des conjonctures du moment?

\section{Conclusion}

Le peu de prises, théoriques et pratiques, qu'eurent les sciences du travail françaises du début du XXe siècle sur les mutilés de guerre et leur sort témoigne des faiblesses et des présupposés de ces sciences. L'irrésolution des problèmes posés par la prise en compte des facteurs psychologiques dans la mesure de l'activité, la valeur jamais interrogée qui est attribuée à l'activité continue et endurante, la négligence scientifique des assises socio-économiques du travail sont autant de zones d'ombre de ces sciences du travail que la singularité pathologique des mutilés révèle. Cette singularité creuse en effet les différences possibles entre santé, possibilité d'activité et de travail qu'Imbert, Lahy et Amar tentent d'unir. Des brouillages, des emballements peuvent survenir. Des formes 
socio-économiques conditionnent une part de l'identité recherchée entre ces trois notions, alors que les mutilés, par impotence, par fatigue ou par déprime, ne cessent de se confronter aux excès possibles, à l'indifférence, au rejet, en tous cas à l'inadaptation du monde du travail à ce qu'ils sont. En théorie comme en pratique, en réalité comme en projet, on ne s'attarde guère sur le sort des invalides au travail. À eux la charge de s'adapter, sans modèle ni repères, s'ils le désirent ou s'ils n'ont pas le choix. Les mutilés de guerre interrogent ainsi, au-delà de leur cas particulier, la teneur des anthropologies positives du travail, qui en promettent l'aménagement en même temps qu'elles affirment l'existence d'une spontanéité à l'activité. En quoi les conditions du travail peuvent-elles échapper à l'exercice de contraintes multiples ? En quoi est-il possible et souhaitable que tous travaillent? Telles sont les questions qui se posent dans la confrontation des sciences psychophysiologiques du travail au cas des invalides, où s'ouvre le problème redoutable du rapport entre science des corps et science politique.

CAMUS Jean, «Placement et rééducation des grands infirmes par lésion des centres nerveux », in Paris Médical, J. B. Baillère et Fils, 1917, n²5, juillet 1917, p.1-5

\section{BIBLIOGRAPHIE}

Emplois réservés aux invalides, veuves et orphelins de guerre, ainsi qu'aux militaires, engagés et rengagés, Volume mis à jour à la date du 31 juillet 1933, Paris, Charles-Lavauzelle et Cie, 1933.

Union des mutilés rééduqués (École de l'Union des colonies étrangères en France), n84, mai 1929, Paris, Société Française d'imprimerie.

AMAR Jules, Le moteur humain et les bases scientifiques du travail professionnel, Paris, Dunod, 1914.

AMAR Jules, L'organisation physiologique du travail, Paris, Dunod et Pinat Éditeurs, 1917.

AMAR Jules, La prothèse et le travail des mutilés, Paris, H. Dunod et E. Pinat Éditeurs, 1916.

AUDOUIN-ROUZEAU Stéphane, Quelle histoire, un récit de filiation (1914-2014), Paris, EHESS/

Gallimard/Seuil, 2013.

BITTARD A.-L., Les écoles de blessés, pensions, prothèses, apprentissage, placement, Paris, Alcan, 1916.

BOURILLON Maurice, Comment rééduquer nos invalides de la guerre. L'assistance aux estropiés et aux mutilés en Danemark, Suède et Norvège, Paris et Nancy, Berger-Levrault, 1916.

CAMUS Jean et VALLÉE Charles, «L'école de rééducation des grands infirmes du système nerveux aux Invalides de Paris (L'Union des Colonies étrangères) ", Revue interalliée pour les questions intéressant les mutilés de guerre, Paris, Imprimerie Chaix, n², juin 1918, p.129-139.

CANGUILHEM, Georges, « Cours de philosophie générale et de logique 1942-1943 », Résistance, philosophie biologique et histoire des sciences 1940-1965, Paris, Vrin, 2015, (Euvres complètes, tome IV).

CANGUILHEM, Georges, Le normal et pathologique, Paris, PUF, 1984.

CANIVEZ, A., La Rééducation professionnelle des mutilés de guerre, Paris, UNMR, 1923. 
DAUSSAT Charles, Expertise des professions. Les mutilations. Physiothérapie. Indemnisation. Législation étrangère. CEuvres d'assistance. Prothèses. Emplois réservés. Travail et salaire. Apprentissage. Répertoire médical des professions et industries avec album de 73 figures, Paris, Vigot Frères Éditeurs, 1925.

HIRSCHFIELD Gustave, CARLE Marius (Dr), Hirschfield, G. et Carle, M., Ville de Lyon. Une école de rééducation professionnelle des grands blessés de la guerre. Tourvielle, par Gustave Hirschfeld, Précédé de considérations sur l'organisation d'une école de blessés, par le Dr M. Carle, Préface par M. Édouard Herriot, Paris, Berger-Levrault, 1917.

IMBERT Armand, Rôle de la physiologie dans les conflits entre le capital et le travail, Paris, Imprimerie Nationale, 1909 (Extrait du Bulletin des sciences économiques et sociales du comité des travaux historiques et scientifiques, 1907).

JEANBREAU Émile, L'école d'apprentissage des éduqués et accidentés de Charleroi, Montpellier, Imprimerie générale du midi, 1910.

LAHY Jean-Maurice, « L'organisation du travail chez les combattants », La Grande Revue, Paris, 1915, p.297-318.

LAHY Jean-Maurice, « Comment peut-on déterminer scientifiquement la fatigue chez les ouvriers qui n'accomplissent pas d'efforts musculaires ", Das Österreichische Sanitätswesen, 30. Jahrgang 1918, Beiheft zu Nr. 1-26.

LAHY Jean-Maurice, Système Taylor et la physiologie du travail professionnel, Paris, Gauthier-Villars et Cie, 1921.

LE BIANIC Thomas et VATIN François, « Armand Imbert, (1850-1922), la science du travail et la paix sociale », Travail et emploi, $\mathrm{n}^{\circ} 111$, juillet-septembre 2007.

LESCENE René, La rééducation professionnelle des amputés des deux mains, Bordeaux, Imprimerie J. Géroude \& Frère, 1921 (Thèse pour le doctorat en médecine).

MAZEL Pierre, La main d'œuvre nationale après la guerre, Paris, Larousse, 1917.

RABINBACH Anson, Le moteur humain, l'énergie, la fatigue et les origines de la modernité, Paris, La Fabrique, 2004.

RAZOUS Paul, Blessés de guerre, prothèses et rééducation professionnelle, l'office national et les comités départementaux et le fonctionnement des œuvres, Paris, Berger-Levrault, 1919.

TOURNADE A., La rééducation professionnelle des mutilés de la guerre; rôle du service de santé, Paris, L. Fournier, 1917.

\section{NOTES}

1. Pour une histoire générale de cette science du travail française, voir par exemple RABINBACH Anson, Le Moteur humain, l'énergie, la fatigue et les origines de la modernité, Paris, La Fabrique, 2004. 2. Suivant l'expression alors usitée, qui donne son titre au célèbre ouvrage de Jules Amar, Le Moteur humain et les bases scientifiques du travail professionnel, Paris, Dunod, 1914.

3. AMAR Jules, L'organisation physiologique du travail, Paris, Dunod et Pinat Éditeurs, 1917, p.231-232. Idem dans sa conférence éditée en 1916, La prothèse et le travail des mutilés, Paris, H. Dunod et E. Pinat Éditeurs, 1916, p.4.

4. LAHY Jean-Maurice, Le Système Taylor et la physiologie du travail professionnel, Paris, GauthierVillars et Cie, 1921, p.213 (première édition en 1916). 
5. AMAR Jules, L'organisation..., op. cit., 1917, p.354, ainsi que pour les autres références du paragraphe.

6. AMAR Jules, ibidem, p.261ss.

7. Sur ces points, voir par exemple : CANIVEZ A., La rééducation professionnelle des mutilés de guerre, Paris, UNMR, 1923, p.5.

8. RAZOUS Paul, Blessés de guerre, prothèses et rééducation professionnelle, l'office national et les comités départementaux et le fonctionnement des œuvres, Paris, Berger-Levrault, 1919, p.19.

9. LESCENE René, La rééducation professionnelle des amputés des deux mains, Bordeaux, Imprimerie J. Géroude \& Frère, 1921 (Thèse pour le doctorat en médecine), p.27.

10. Voir successivement CAMUS Jean, «Placement et rééducation des grands infirmes par lésion des centres nerveux ", Paris Médical, J. B. Baillère et Fils, n²5, Juillet 1917, p.1-5; puis CAMUS Jean et VALLÉE Charles, "L'école de rééducation des grands infirmes du système nerveux aux Invalides de Paris (L'Union des Colonies étrangères) ", in Revue interalliée pour les questions intéressant les mutilés de guerre, $\mathrm{n}^{\circ} 2$, juin 1918, Paris, Imprimerie Chaix, 1918, p.129-139.

11. GOURDON J., DIJONNEAU H. et THIBEAUDEAU J., Rendement professionnel des malades de guerre, Paris, Gustave Doin Éditeur, 1923, p.26 et 44.

12. CANIVEZ A., La rééducation..., op. cit., 1923, p.67.

13. Voir par exemple IMBERT Armand, Rôle de la physiologie dans les conflits entre le capital et le travail, Paris, Imprimerie Nationale, 1909 (Extrait du Bulletin des sciences économiques et sociales du comité des travaux historiques et scientifiques, 1907).

14. Das Österreichische Sanitätswesen, 30. Jahrgang 1918, Beiheft zu Nr. 1-26.

15. AMAR Jules, L'organisation..., op. cit., 1917, p. 65-116 ; LAHY Jean-Maurice, Le Système Taylor..., op. cit., 1921, p.149-169.

16. AMAR Jules, ibidem, p.176.

17. LAHY Jean-Maurice, ibidem p.174ss.

18. LAHY Jean-Maurice, ibid., p.180.

19. Sur ce point, voir CANGUILHEM Georges, Le Normal et pathologique, Paris, PUF, 1984, par exemple p.132 ou LE BLANC Guillaume, Les Maladies de l'homme normal, Paris, Vrin, 2007.

20. CANGUILHEM Georges, "Cours de philosophie générale et de logique 1942-1943 », Résistance, philosophie biologique et histoire des sciences 1940-1965, Paris, Vrin, 2015, (Euvres complètes tome IV).

21. BOURILLON Maurice, Comment rééduquer nos invalides de la guerre. L'assistance aux estropiés et aux mutilés en Danemark, Suède et Norvège, Paris et Nancy, Berger-Levrault, 1916, p.149.

22. JEANBREAU E., L'école d'apprentissage des éduqués et accidentés de Charleroi, Montpellier, Imprimerie Générale du midi, 1910, p.6.

23. BITTARD, A.-L., Les écoles de blessés, pensions, prothèses, apprentissage, placement, Paris, Alcan, 1916, p.130.

24. Les avis sont partagés pendant la guerre au sujet des écrits de Jules Amar, notamment Le moteur humain et les bases scientifiques du travail professionnel, paru pour la première fois en 1914 . André Tournade l'évoque sans appliquer effectivement ses méthodes dans La rééducation professionnelle des mutilés de la guerre; rôle du service de santé, Paris, (L. Fournier, 1917). Carle le juge inutile et argumente son rejet en renvoyant à une thèse de médecine de Vallée, La mesure des impotences (voir Ville de Lyon. Une école de rééducation professionnelle des grands blessés de la guerre. Tourvielle, par Gustave Hirschfeld, Précédé de considérations sur l'organisation d'une école de blessés, par le Dr M. Carle, Préface par M. Édouard Herriot, Paris, Berger-Levrault, 1917, p.31.)

25. AMAR Jules, L'organisation..., op. cit., 1917, p. 338.

26. Sur ce double rejet du travail des mutilés, par le marché de l'emploi et par les mutilés euxmêmes, voir : MONTES Jean-François, 1915-1939, (re)travailler ou le retour du mutilé : une histoire de l'entre-deux-guerres, Paris, Rapport de recherche pour l'office National des Mutilés et Réformés, 1991. 
27. AMAR Jules, L'organisation..., op. cit, 1917, p.329 ; LAHY Jean-Maurice, Le Système Taylor..., op. cit. , p.164.

28. LAHY, ibidem, p.154.

29. AMAR Jules, L'organisation..., op. cit.,1917, p.334.

30. AMAR Jules, ibidem, p.330.

31. MAZEL Pierre, La main d'œuvre nationale après la guerre, Paris, Larousse, 1917.

32. DAUSSAT Charles, Expertise des professions. Les mutilations. Physiothérapie. Indemnisation. Législation étrangère. Euvres d'assistance. Prothèses. Emplois réservés. Travail et salaire. Apprentissage. Répertoire médical des professions et industries avec album de 73 figures, Paris, Vigot Frères Éditeurs, 1925, p. 85-86.

33. MONTES Jean-François, 1915-1939..., op. cit., p.44.

34. Union des mutilés rééduqués (École de l'Union des colonies étrangères en France), n84, mai 1929, Paris, Société Française d'imprimerie, p.7.

35. Voir par exemple, AUDOUIN-ROUZEAU, Stéphane, Quelle histoire, un récit de filiation (1914-2014), Paris, EHESS/Gallimard/Seuil, 2013.

36. Voir par exemple la liste III, p.103-166 du volume Emplois réservés aux invalides, veuves et orphelins de guerre, ainsi qu'aux militaires, engagés et rengagés, Volume mis à jour à la date du 31 juillet 1933, Paris, Charles-Lavauzelle et Cie, 1933.

37. AMAR Jules, L'organisation..., op. cit., 1917, p.340ss.

38. LAHY Jean-Maurice, Le Système Taylor..., op. cit., 1921, p.189-193.

39. LAHY Jean-Maurice, ibidem, p. 187.

40. Voir Le BIANIC Thomas et VATIN François, "Armand Imbert, (1850-1922), la science du travail et la paix sociale », Travail et emploi, $\mathrm{n}^{\circ} 111$, juillet-septembre 2007, p.7-19.

41. LAHY, Jean-Maurice, "L'organisation du travail chez les combattants", La Grande Revue, Paris, 1915, p.297-318.

\section{RÉSUMÉS}

Les problèmes posés par la remise au travail des invalides de la Première Guerre Mondiale pointent certaines faiblesses des sciences du travail de l'époque, telles qu'essaient de les élaborer Armand Imbert, Jean-Maurice Lahy et Jules Amar sur la base de calculs physio-psychologiques. De manière étonnante, on reconnaît qu'une partie des « moteurs humains » que sont les mutilés de guerre ne pourra pas trouver à s'employer. Plus précisément, l'évaluation de l'endurance des anciens combattants, la modélisation de leur récupération fonctionnelle possible, leur insertion dans l'organisation collective des usines font difficulté. Au-delà des incertitudes, aveuglements, et oublis scientifiques ou politiques des promoteurs d'une science physio-psychologique du travail, le cas des mutilés de guerre permet de montrer que leurs visées ne trouvent leur impulsion ni dans un positivisme, ni dans une politique, mais dans une anthropologie où l'activité spontanée de l'homme peut être la source d'un travail productif non contraint.

The problems posed by the return to work of the disabled in the First World War point to certain weaknesses of the labor sciences of the time, as Armand Imbert, Jean-Maurice Lahy and Jules Amar tried to elaborate them on the basis of physio-psychological calculations. Surprisingly, it is recognized that some of the "human engines" that are the mutilated warriors will not find employment. More specifically, the assessment of the endurance of veterans, the modeling of 
their possible functional recovery, and their insertion into the collective organization of the factories are difficult. Beyond the uncertainties, blindness, and scientific or political forgetfulness of the promoters of a physio-psychological science of work, the case of the war disabled makes it possible to show that their aims do not find their impulse either in positivism or in politics, but in an anthropology where the spontaneous activity of man can be the source of unforced productive work.

\section{INDEX}

Mots-clés : histoire des techniques, travail, invalidité, France, Première Guerre Mondiale Index chronologique : Époque contemporaine Index géographique : France

Keywords : history of technology, labor science, capacity assessment, disability, France, World War I

\section{AUTEUR}

\section{STÉPHANE ZYGART}

Né en 1977, docteur en philosophie, enseignant à l'université de Lille dont les recherches portent sur les rapports entre médecine et société, d'un point de vue philosophique, politique et éthique. Thèse sur les normes médico-sociales contemporaines en cours de publication. 